\title{
Exploring Academic Voice in Multimodal Quantitative Texts
}

\author{
ROBERT PRINCE AND ARLENE ARCHER
}

\begin{abstract}
Research on students' academic literacies practices has tended to focus on the written mode in order to understand the academic conventions necessary to access Higher Education. However, the representation of quantitative information can be a challenge to many students. Quantitative information can be represented through a range of modes (such as writing, visuals and numbers) and different information graphics (such as tables, charts, graphs). This paper focuses on the semiotic aspects of graphic representation in academic work, using student and published data from the Health Science, and an information graphic from the social domain as a counterpoint to explore aspects about agency and choice in academic voice in multimodal texts. It explores voice in terms of three aspects which work across modes, namely authorial engagement, citation and modality. The work of different modes and their inter-relations in quantitative texts is established, as is the use of sources in citation. We also look at the ways in which credibility and validity are established through modality. This exploration reveals that there is a complex interplay of modes in the construction of academic voice, which are largely tacit. This has implications for the way we think about and teach writing and text-making in quantitative disciplines in Higher Education.
\end{abstract}

\section{Exploring academic voice in multimodal quantitative texts}

Texts in quantitative disciplines are constructed using language, images such as information graphics (charts, maps or diagrams), and mathematical notation which together form the repertoire of 
quantitative semiotic resources. Halliday (1978) argues that mathematical language typically draws on a variety of 'language' types, redefines existing words rather than coining new ones, and uses a high degree of nominalisation. He refers to this as a 'mathematical register'. The notion of a mathematical register is useful as it enables a 'critical interrogation of assumptions of the purity or semantic distinctiveness of mathematical language' (Chapman and Lee 1990:282). It is useful to analyze how mathematical notation, written language and visuals such as information graphics work together, especially in terms of fulfilling specific functions in academic argument in particular contexts (O'Halloran 1999, 2009). These functions - working across and between modes - include drawing inferences, perceiving logical relationships, evaluating cause and effect, ordering and sequencing, and constructing hypotheses. We argue that there is a need to develop a pedagogy that takes into account these functions of academic argument.

In this paper, our investigation focuses on meaning-making across writing and information graphics in quantitative texts. We use the term 'information graphics' to encompass charts, graphs, maps, diagrams and tables. Their primary function is 'to consolidate and display information graphically in an organized way so a viewer can readily retrieve the information and make specific and/ or overall observations from it' (Harris 1999:198). Engineering and architectural drawings are not included under the classification of information graphics. We focus our exploration specifically on multimodal texts that employ information graphics to identify and discuss academic voice.

'Voice' is an elusive concept, difficult to define, yet definitions abound. It has come to be associated with 'authorial identity' (Ivanič 1998) and 'authorial presence' (Hyland 2001). Voice is about identity and presentation of the self in texts. Although voice is a concept usually associated with the mode of writing, we use it to refer to the way an author establishes presence in a multimodal text through the choice and use of semiotic resources, as well as positioning in relation to sources and audience. Blommaert (2005:68) refers to voice as 'the capacity to create favourable conditions for a desired uptake' using the appropriate semiotic resources to do so. What is useful about this view is that it emphasizes voice as subject to contextual conditions which are necessarily located within larger patterns of inequality and 
power relations. This is clearly the case with academic discursive conventions which have often been seen as 'gatekeepers' in terms of student access (Archer, Frith and Prince 2002, Lea and Street 1998, Lillis 2001, Street and Baker 2006). Exploring the ways in which academic voice operates in different contexts and disciplines is important in terms of making these conventions explicit in order to 'master' them, or at least engage with them on a conscious level.

\section{A social semiotic approach to voice in quantitative texts}

Our approach to exploring voice is multimodal social semiotics where meaning is seen to be context-dependant and meaning-making is seen as a social practice (Martinec and Van Leeuwen 2008, Van Leeuwen and Jewitt 2001, Kress 2010, Jewitt 2009). The assumption underpinning this approach is that meaning-making is embedded in context. Also, meaning is understood to be made through the selection and configuration of modes in texts and through the interests of the sign-maker in a particular context (Jewitt 2009:15). According to Halliday (1978), every sign performs three kinds of function. The ideational function represents the world, concepts and processes. The interpersonal function indexes the stance that the meaning-maker is taking towards audiences and the represented context. The textual function refers to the capacity to form coherent complexes of signs or texts. So, for instance, an information graphic represents a state of affairs, a relationship between abstract 'participants', and it also indicates a particular relationship with an audience. These ideational and the interpersonal aspects are realized through the textual organization or composition of the graphic.

A social semiotic approach can help us to understand the ways in which writing and information graphics work together in meaningmaking. Each mode in a multimodal text can realize a range of different functions or communicative work. So, different aspects of meaning can be carried in different ways by each of the modes. The information graphic could, for instance, serve as elaboration on the writing. It could be used as evidence in an argument, it could be a part of an argument (the proposition, for instance), or it could constitute 'restatement' in a different mode. Sometimes, the communicative work of different modes in a text can complement each other, but they can also contradict each other. Where different modes realize different aspects in a complementary way, textual coherence may be achieved. However, where there is a disjuncture between the 
messages of the modes in a text, coherence could be compromised (Archer 2010). Recognizing the communicative work that different modes are performing in a text is important for the teaching of academic voice and argument in quantitative disciplines. Some modes are better than others for certain kinds of representational work (Kress and Van Leeuwen 2006). It can be difficult to multiply quantities in words, for example. Writing is perhaps less precise at giving descriptions of natural phenomena in which matters of degree or quantitative variation are important (Lemke 1998). It is difficult, for example, to describe movement through space in words, to indicate the changing speed and the shape of the movement at the same time. Lemke (2002:8) calls this 'meaning-by-degree', like size, shape, distance, proportion, intensity, speed, pressure, density, rates of change, and he argues that these kinds of meaning are often best represented by mathematical notation rather than language.

In this paper, we are interested in the notion of 'voice' which falls in the realm of the interpersonal metafunction in texts. We examine the integration of information graphics and writing in terms of authorial engagement, and look at the ways in which citation is used to construct voice. Authorial engagement and citation signal authorial positioning in relation to a particular research community or readership. We also look at the produced shared credibility of a representation. This is an adaption of the broad framework developed in Archer (2013) and used to explore the realization of voice across images and writing in students' texts in first year History and Theory of Architecture. We argue that the way three aspects come together relates to academic voice, namely authorial engagement, citation and modality.

To illuminate the ways in which the above three aspects function to establish academic voice, we firstly look at an extract from a PhD thesis in medical virology in the Health Sciences. The aim of the study was to investigate the therapeutic role of two vaccinations (the 'humanized recombinant vaccinia virus complement control protein' (hrVCP) and the 'vaccinia infected mammalian cell derived authentic VCP') in Ischemia/Reperfusion (I/R) injury of the kidney in rats. The three groups are the treated rats, untreated rats and the 'sham' group which comprises rats which have not been injured or treated. An extract from the thesis appears in figure 1 below. 


\section{Serum Creatinine and BUN}

As shown in Figure 4 below, there was considerable difference (1.2-fold \pm 0.127 ) in the serum creatinine levels between the untreated injured (PBS) group and the VCP/hrVCP treatment group, suggesting that the VCP improved renal function and enabled the renal system to excrete the metabolic waste better than the equally injured animals in the untreated group. The sham group displayed the lowest serum creatinine levels as expected. However, there was no statistically significant difference in the serum urea and creatinine levels among any of the groups.

Figure-4: Mean Serum creatinine concentration after 24hrs I/R injury. Bars represent standard errors of sample mean (mean \pm SEM).

Comparison of serum creatinine conc. following $I / R$ injury



Interestingly, one additional, bilaterally clamped, animal showed a 2.9-fold rise in serum creatinine and a 25.3-fold rise in serum BUN levels after 24 hours of I/R injury compared to its baseline concentrations suggesting that acute renal failure (ARF) was induced in this animal.

\section{Figure 1: Extract from a $\mathrm{PhD}$ thesis in medical virology in the} Health Sciences

Having presented the data and the framework for analysis, we now investigate academic voice in the above text in more detail focusing on authorial engagement, the use of citation and the construction of modality. 


\section{Authorial engagement, agency and choice}

One aspect of academic voice is authorial engagement, namely an author's degree of intimacy or remoteness, and the ways they represent themselves in the discourse' (Hyland 1999:101). Authorial engagement concerns the extent to which authors choose to engage with their audiences and subject matter. Authorial engagement is actualized in writing and information graphics through different mode-specific conventions.

In writing, authorial engagement can be revealed through attitude markers and use of modifiers, such as 'there was considerable difference ...' Interestingly, this is standard expression of a quantitative idea. It can also be established through the choice of pronouns, active or passive voice, rhetorical questions and even through the visual design of the writing such as typographical choices, use of punctuation, layout and use of white space (Archer 2013).

In graphic representation authorial engagement is expressed through choice such as the type of representation, the composition of the representation and the relation between the information graphic and the writing. The type of information graphic chosen for a particular purpose is important as there are a number of ways of presenting summative numeric information, numeric ranges and percentages. Broadly speaking, bar charts are able to compare quantities, pie charts are useful to show proportions of the whole, line graphs can show quantities over time, scatter plots are useful in exploring meaningful relationships between variables, venn diagrams are able to represent relationships where circles are used to show groups and how they overlap.

In figure 1, the student has chosen a bar chart with confidence intervals to represent his data graphically. The bulky size and shape of the bar certainly gives more visual weight to the argument than a single point does. Here the blue bars represent the mean Serum creatin concentration which is a single value, and the confidence interval is represented by the interval (mean - SEM, mean + SEM) which is set on a grey background. In the written text, there is a specific use of values (1.2-fold \pm 0.127 ), but the graphical representation does not allow for the verification of this value. In the information graphic, one has to estimate the value due to the lack of lines and the fact that the value is not indicated on the point. Tufte's 
(2001) argument for keeping the Data -Ink Ratio high would have encouraged the use of a single point to represent each of the means rather than using bars to represent this. The ink required to draw a bar is more than what is required to draw a dot. The Data-Ink Ratio argument would also favour the removal of the grey background colour of the chart.

The student could have chosen to represent the information in a table format, rather than a bar chart. According to Tufte (2001:178), tables are a good way to show exact numerical values and are preferable to graphics for small data sets. If the student chose to represent the information in a table, this would impact on the type of information possible to display. For instance, displaying the sample size would have been more likely in a table; its absence in the bar chart is not so obvious. A table display would also have allowed verification of the claims about the difference between the serum creatine levels of the untreated injured group and the treatment group. However, increasingly in both popular texts and academic discourse, graphic representations tend to be regarded as more weighty and scientific, and to have more rhetorical import than tables, which are sometimes considered to be a simplified representational form. In thinking about academic voice and the teaching of multimodal textual construction, awareness about the importance of choice of information graphic for a particular purpose and audience is important, as are the conventions and functions of the graphic in a particular context.

In quantitative texts, authorial engagement is also realized through layout and the composition of the information graphic. The chart in figure 1 comprises blue bars on a grey background which is the default design in Excel. There are a host of other design choices that the student could have made around this particular chart, such as using different colours for different bars for ease of comparison. The wide spacing in the chart in figure 1 creates a clear distinction between the different groups that are being compared. However, the choice of wide spacing tends to compromise the clarity of argument of this chart because it is more difficult to compare between the groups at a glance. Perhaps using the default design in Excel, to produce this chart, rather than changing the spacing, and also not including horizontal lines to facilitate ease of reading, signals low authorial engagement. These representational choices weaken academic voice and authorial agency to an extent in the text. They 
may, however, also signal larger patterns of inequality and power relations, such as, not having the resources or the sense of agency to exert changes on a dominant form.

We have argued that authorial engagement is expressed through choice, namely the type of representation and the composition of the representation. In creating information graphics, we need to be aware of the full range of representational resources in order to make appropriate selections for the particular domain in which we are operating, both in terms of content and argument or voice. As a counterpoint to the representational choices discussed above, it is worth looking at information graphics which are composed very differently, such as those produced by McCandless (2009) or showcased in Cooke (2013). Of course, representational choices depend on context - aptness for purpose and audience. However, looking at different kinds of information graphics produced for different contexts may raise questions that enable us to reflect on authorial voice in academic contexts. As Thesen describes it, "voice, like language, is never neutral; it is always in tension between pulls towards convention (centripetal forces) and pushes away from the centre towards more hybrid, experimental and open forms" (Thesen 2014: 6). This may be the case in an information graphic such as figure 2 . 


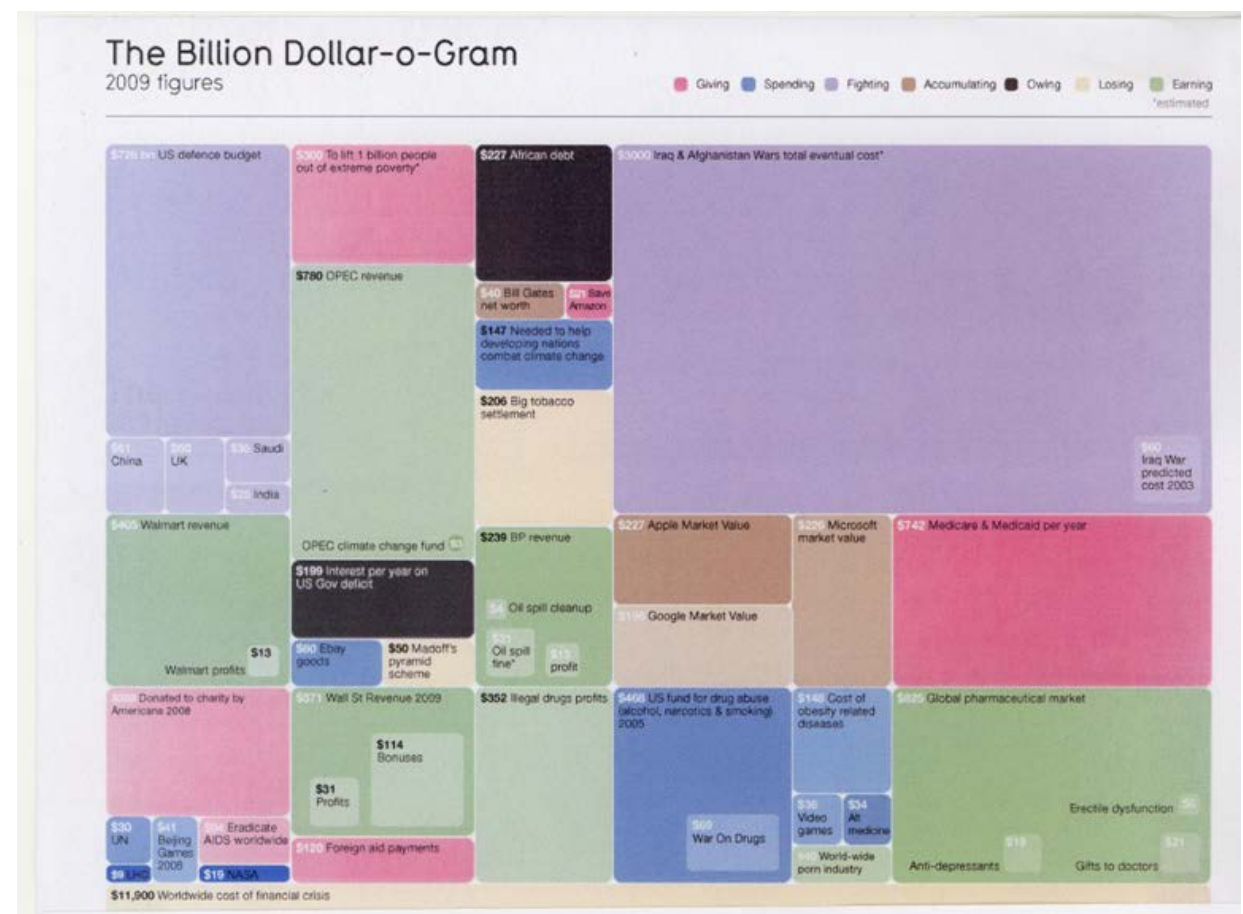

Figure 2: The Billion-Dollar-O-Gram (McCandless 2009:10)

Figure 2 is a variation of a mosaic plot. McCandless (2009:10) calls it the 'billion-dollar-o-gram' a title that signals the tongue-incheek social commentary of the graphic. Here colour is used to perform the ideational function of the text in order to contrast what the chart names American spending, earning, potential giving, fighting and losing. From the diagram it is clear that three quarters of the annual defence budget is spent on the Iraq war. McCandless compares the US defence budget to a large company's earnings and to other countries' budgets (the US defence budget can include China's and Russia's defence budget, for instance). Because this information graphic is spatially rather than linearly organized, one can tell many stories from the information, not necessarily in any particular order. However, by placing the US defence budget in the upper left hand corner, a valorized space where many left-to-right readers would focus their attention first, a particular relationship of this category to the other categories is encouraged. The choice of items to compare reflects the arguments the author wishes to make. His is a social agenda: to contrast the expenses of war with the expenses of developmental issues. These kinds of information 
graphics are prevalent in our era of big data. As information moves fast, they are able to offer us quick bursts of information on the environment, politics, social issues, health, arts and culture. Whilst recognizing that these kinds of information graphics are embedded in socio-political discourses and realized in journalistic genres, they are useful in demonstrating a different kind of authorial engagement and power-differential than the student-produced graphic.

By calling his book Information is Beautiful, McCandless (2009) draws attention to the 'aesthetic' aspect of the production of information graphics. There are design choices to be made here in terms of size, shape, colour and composition in order to represent a particular argument to a particular audience in the most apt way. These choices are always expressions of ideology and power - what choices were made and for what purposes? The representational choices made here may not be appropriate in an academic environment, and may, in fact, weaken academic voice, whereas they function well in popular journalistic genres. This highlights a tension between aesthetics and functionality in information graphics which may have implications for academic voice. Understanding the relation between agency and constraints is crucial for exploring how we construct authorial voice in the texts we produce in particular contexts.

\section{Use of citation and sources in constructing academic voice}

The use of citation is crucial to the construction of academic voice. Citation in both writing and information graphics involves appropriating a source into the argument and using the voices of others to negotiate a position in a particular discourse community (Swales 2004). Choices about the integration of sources include the selection of material from the source and the form of the citation, as well as critical evaluation or some kind of framing. In writing, citation can take the form of quoting, paraphrasing or generalizing from several sources. How the source is foregrounded or backgrounded is of importance for academic voice. The two statements below illustrate this point:

- Vygotsky (1967) indicates that language is the most important tool for mastering mental processes. 
- Language is the most important tool for mastering mental processes (Vygotsky 1967).

In the first statement, the source is prioritized and, in the second, the argument is foregrounded. Although these citation conventions are based in the written mode, it could be interesting to explore whether there is an equivalent in information graphics. Perhaps it is the question itself that is more interesting than the answer, in that it opens up a new way of thinking around the use of sources in constructing voice. We would argue that the placing of the in-text reference in the information graphic is of importance. The source could be more foregrounded if placed in the label than the caption, for instance. The way in which the source is introduced is also of importance. For instance, the words 'taken from ...' indicate the graphic is a reproduction or a 'quote' from the original source with all the deferment of authority that this entails. If the source is introduced as 'adapted from ...' it indicates paraphrasing and possibly increased authorial engagement.

There are three options for citation in information graphics. The data could be empirical and thus no reference is necessary as is the case with the student chart in figure 1 . The chart is based on his own data set that is not represented here. A second possibility is the integration of a researcher's own data with cited data. And, thirdly, data can be compiled from multiple sources within one information graphic, in which case the citations are placed beneath each bar in a bar graph. It is when students battle with the conventions around referencing that we realize how invisible and normative they are. For instance, in the caption of a different information graphic in the thesis, the student ends with the following: "The analysis was performed by Dr X, Department of Zoology, UCT using the " $\mathrm{R}$ ” software package'. This is a kind of citation in that it acknowledges the work of a data analyst, other than himself, as well as the software package used. However, it is a hybrid form, somewhere between citation and acknowledgements.

We suggest that the placement of the citation in an information graphic may have implications for academic voice, as well as the degree of adaptation in terms of 'paraphrasing' or re-working the graphic to best suit the particular argument. It is worth noting that 'multimodal text-production through copy-and-paste is an affordance of the digital medium that has profound consequences in the ways 
texts are composed' (Adami 2012:131). Copy-and-paste makes direct quoting easier than paraphrasing, in all modes. In sum, the choice of source, the re-working of the source in terms of paraphrasing, and the integration of the source, all have implications for academic voice.

The last aspect of academic voice we would like to look at is the way in which credibility is established through the use of modality markers.

\section{Modality: the representation of degrees of certainty}

Modality refers to the author's expressed commitment to producing truth effects, in other words, the produced shared credibility of a representation. In academic writing, tentative modality is often the norm and this is realized through discourse markers such as hedging and emphatics (Hyland 1999:104). Hedges indicate the writer's decision to withhold complete commitment as in 'suggesting that the VCP improved renal function' (see figure 1), whereas emphatics construct certainty: 'The sham group displayed the lowest serum creatinine levels as expected' (see figure 1). Prince and Archer (2008) argue that information graphics tend to carry a lot of credibility in academic texts as the assumptions underlying the numbers are generally hidden and numerical representations are often regarded as more factual and objective than other kinds of evidence.

What does modality look like in information graphics and multimodal quantitative texts? The type of information graphic chosen can lend more authority to the numbers. For instance, the use of a table in a text may lower modality where an information graphic may heighten modality in a particular scientific domain. Modality, in a social semiotic approach to information graphics, is primarily concerned with representations of degrees of uncertainty and the validity claims associated with the graphic.

An important aspect of modality in quantitative texts is the representation of uncertainty. In statistics, uncertainty is often dealt with by interpreting the confidence intervals. In the written text in figure 1 , the author uses the configuration 1.2 -fold \pm 0.127 to hedge the size of the difference. In other words, the size of the difference is somewhere between 1.073 and 1.327. In information graphics, uncertainty about and confidence in a 'point statistic' are often provided through the determination and use of an 'interval statistic' alongside it. In this case, the mean and the standard error of the mean 
(SEM) are used to create a confidence interval (Mean - SEM, mean + SEM). In the information graphic in figure 1, the bars in the chart are used to represent the means and the confidence intervals are used to convey both the uncertainty about and the confidence in the means, perhaps akin to hedging in writing.

Confidence intervals represent the range of values possible and allow us to present our findings in a more tentative way which can be likened to tentative modality in writing in academic discourse.

Results from studies such as the one represented in figure 1 one often vary through chance alone. The aim was to investigate the therapeutic role of two vaccinations in the healing of Ischemia/reperfusion (I/R) injury in the kidney of rats. These kinds of studies differ in terms of the subjects who are included, and the ways in which these specific subjects react to therapeutic interventions. Even when everything possible is held constant, there will still be some random variations. For this reason, tools are needed to assess whether the differences between treated and untreated groups of subjects are significant, or just due to chance. Historically, p-values were used to represent statistical significance, but increasingly confidence intervals are serving this purpose. A p-value is calculated to assess whether trial results are likely to have occurred simply through chance. A confidence interval is calculated to provide a measure of treatment effect and shows the range within which the treatment effect, subject to a range of assumptions, is likely to lie. P-values provide a cut-off point, beyond which it can be asserted that the findings are 'statistically significant'. Statistical significance, however, does not necessarily mean that the effect is 'real', since by chance alone, about one in 20 significant findings will be spurious. Confidence intervals tell us the range of possible values. As such, they aid with the interpretation of clinical trial data by putting upper and lower bounds on the likely size of any effect. A confidence interval that embraces the value of no difference between treatment groups indicates that the treatment under investigation is not significantly different from the control group. Standard error bars indicate the error or uncertainty in a reported measurement. For sufficiently large numbers the standard error bars can be doubled to get, approximately, the $95 \%$ confidence intervals and the standard error bars give approximately a $68 \%$ confidence interval, so that in about two thirds of cases standard error bars capture the mean. 
In the example mentioned earlier, the student uses standard error bars rather than confidence intervals. The statistical analysis to be used is described by the student as 'mean \pm SEM of $n$ independent experiments'. In many disciplines, including medicine and psychology, there continues to be debate about the choice of these statistical tools to represent uncertainty (Fidler et al 2004). In particular, confidence intervals are preferred to both p-values and SE bars (Cumming and Finch 2005). Cumming and Finch (2005:170) argue that many researchers 'have important misconceptions about confidence intervals' and that 'there are few accepted guidelines as to how confidence intervals should be presented or discussed'. The challenges in determining, reporting and discussing the p-values and standard error bars which the student experienced during this stage of his study exemplified Cumming and Finch's views.

In some cases in information graphics, colour can be used to represent degrees of certainty. In many visuals, use of colour is often seen as indexical of modality, enabling a representation such as a photograph to be more or less 'true to life' in a particular domain through the choice of colour and colour saturation. In information graphics, however, colour is more conceptual than interpersonal, often fulfilling the ideational metafunction. In the 'billion-dollar-ogram' in figure 2, colour is used to differentiate between different categories in order to compare them. Here the conventions around colour usage are specific to this particular graphic. However, there are some conventions around colour that work across particular types of graphics. For instance, in mosaic plots over the last 15 years, colour and colour saturation have come to represent size. A mosaic plot is an area-proportional visualization of typically observed frequencies. It is composed of tiles corresponding to the cells, which are created by vertical and horizontal splits of a square. The area of each tile is proportional to the corresponding cell entry given the dimensions of previous splits (see figure 3 below). 


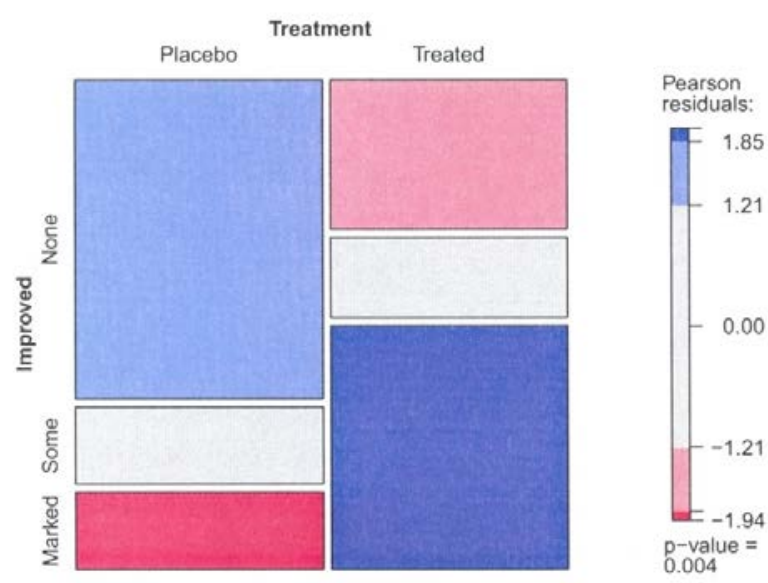

Figure 1: Mosaic plot for the Arthritis data.

Figure 3: Mosaic plot for the Arthritis data (Meyer et al 2005:3)

Here, blue represents a larger than expected number whereas red represents a less than expected number with the associated statistical significance indicated on the scale. In a sense, there is an evaluative element here where blue is positively valued and red more negatively valued. While the area of each tile provides information about the proportion, the colour saturation of the tile provides an indication of the statistical significance of this proportion using 'Pearson residuals'. The Pearson residual is a measure of the difference between the actual proportion in each cell and the proportion expected purely by chance. The grey is used to show that the difference is not statistically significant. So, here 'colour' and colour saturation are used to represent degrees of certainty.

We have shown some of the ways in which modality is constructed and negotiated in quantitative texts through confidence intervals and through the use of colour. The link between modality and academic voice is a complex one to negotiate and usually forms part of the 'unconscious' practices of a particular discipline. It seems important to explore modality and voice with students in more overt ways. This can be done through highlighting the ways of expressing 
degrees of uncertainty or 'hedging' conventions in both information graphics and the written text.

\section{Final comments}

We have presented an analysis of multimodal texts in an attempt to show how academic voice is constructed through an interplay of multiple semiotic resources. Through a discussion of authorial engagement, citation and modality our focus has been on the interpersonal, looking at the ways in which authors insert their presence into a quantitative text. Written language, visual representation, mathematical notation and the representational choices we make (like using a bar rather than a point on a graph) all contribute to academic voice. In composing quantitative academic texts, students need to persuade readers about the relevance and validity of the argument. This involves encoding ideational material and establishing relationships within the discourse community, both through citation and the use of modality conventions (such as using confidence intervals, standard error bars and p-values for representing degrees of certainty). Our exploration reveals that there is a complex interplay of modes in the construction of academic voice, which are largely tacit. This has implications for the way we think about and teach writing and text-making in quantitative disciplines in Higher Education.

In teaching text construction, the notion of drafting and redrafting in writing has much currency. We would like to argue that information graphics could also go through a process of production and reproduction, so that, for instance, the default design in Excel is not the automatic representational choice. We have argued for exploring the full range of available resources in producing information graphics in Higher Education (including those from popular culture) in order to interrogate and strengthen our own design practices. A way of looking at academic voice such as the one explored here could be useful to facilitate awareness and analysis of multimodal texts in order to enable student access to the invisible norms and conventions of quantitative disciplines. It could also facilitate recognition of the social provenance of texts, namely that information graphics are often seen as objective and neutral, rather than ideological. 


\section{References}

Adami, E (2012) The Rhetoric of the Implicit and the Politics of Representation in the Age of Copy-and-Paste, Learning, Media and Technology, vol 37, no 2, pp 131-144. doi: http://dx.doi.org/10.1080/17439884.2011.641567

Archer, A (2013) Voice as Design: Exploring academic voice in multimodal texts in Higher Education, in M Bock and $\mathrm{N}$ Pachler, eds, Multimodality and Social Semiosis. Communication, Meaning-Making, and Learning in the Work of Gunther Kress, Routledge, New York and London, pp150 161.

Archer, A (2010) Multimodal Texts in Higher Education and the Implications for Writing Pedagogy, English in Education, vol 44, no 3, pp 201 - 213. doi: http://dx.doi.org/10.1111/j.17548845.2010.01073.X

Archer, A (2006) A Multimodal Approach to Academic 'Literacies': Problematizing the visual/verbal divide, Language and Education, vol 20, no 6, pp 449 - 462. doi: http://dx.doi.org/10.2167/le677.0

Archer, A, Frith, V and Prince, R (2002) A Project-based Approach to Numeracy Practices at University focusing on HIV/AIDS. Literacy and Numeracy Studies. 11, 2. 123 -131.

Blommaert, J (2005) Discourse, Cambridge University Press, Cambridge. doi: http://dx.doi.org/10.1017/CBO9780511610295

Chapman, A and Lee, A (1990) Rethinking Literacy and Numeracy, Australian Journal of Education, vol 34, no 3, pp 277-289. doi: http://dx.doi.org/10.1177/000494419003400305

Cumming, G and Finch, S (2005) Inference by Eye: Confidence intervals and how to read pictures of data, American Psychologist, vol 60, no 2, pp 170-180. doi: http://dx.doi.org/10.1037/0003-066X.60.2.170

Fidler, F, Thomason, N, Cumming, G, Finch, S and Leeman, J (2004) Editors Can Lead Researchers to Confidence Intervals, but Can't Make Them Think: Statistical reform lessons from medicine, Psychological Science, vol 15, no 2, pp 119-126. doi: http://dx.doi.org/10.1111/j.0963-7214.2004.01502008.x

Halliday, MAK (1978) Language as Social Semiotic. The social interpretation of language and meaning, Arnold, London. 
Harris, RL (1999) Information Graphics: A comprehensive illustrated reference, Oxford University Press, New York.

Hyland, K (1999) Disciplinary Discourses: Writer stance in reesearch articles, in CN Candlin and K Hyland, eds, Writing: Texts, processes and practices, Longman, London and New York, pp 99-121.

Hyland, K (2001) Humble Servants of the Discipline? Self-Mention in Research Articles, English for Specific Purposes, vol 20, pp 207-226. doi: http://dx.doi.org/10.1016/S0889-4906(00)00012$\underline{0}$

Ivanič, R (1998) Writing and Identity. The discoursal construction of identity in academic writing, John Benjamins, Amsterdam. doi: http://dx.doi.org/10.1075/swll.5

Jewitt, C, ed, (2009) The Routledge Handbook of Multimodal Analysis, Oxford University Press, Oxon.

Kress, G (2010) Multimodality: A social semiotic approach to contemporary communication, Routledge, Oxon, New York.

Kress, G and van Leeuwen, T (2006) Reading images. The grammar of visual design, Routledge, London.

Lea, MR and Street, B (1998) Student Writing and Faculty Feedback in Higher Education: An academic literacies approach, Studies in Higher Education, vol 23, no 2, pp 157-165. doi: http://dx.doi.org/10.1080/03075079812331380364

Lemke, JL (1998) Multiplying Meaning: Visual and verbal semiotics in scientific text, in JR Martin and R Veel, eds, Reading Science, Routledge, London.

Lemke, JL (2002) Mathematics in the Middle: Measure, picture, gesture, sign, and work, retrieved 17 September 2012 from http://www.jaylemke.com/storage/Math-in-the-Middle2002.pdf.

Lillis, T (2001) Student Writing: Access, regulation, desire, Routledge, London.

Martinec, R and Van Leeuwen, $\mathrm{T}$ (2008) The Language of New Media Design. Theory and practice, Routledge, London and New York.

McCandless, D (2009) Information is Beautiful, Collins, London.

Meyer, D, Zeileis, A and Hornik, K (2005) The Strucplot Framework: Visualizing Multi-way Contingency Tables with vcd, retrieved 11 May 2010 from http://statmath.wu-wien.ac.at/. 
O’Halloran, K (1999) Towards a Systemic Functional Analysis of Multisemiotic Mathematics Texts, Semiotica, vol 124 (1/2), pp 1-29. doi: http://dx.doi.org/10.1515/semi.1999.124.1-2.1

O’Halloran, K (2009) Historical Changes in the Semiotic Landscape. From calculation to computation, in Jewitt, Carey, ed, Routledge Handbook of Multimodal Analysis, Routledge, Oxon and New York, pp 98-113.

Prince, R and Archer, A (2008) A New Literacies approach to academic numeracy practices in Higher Education in South Africa, Literacy and Numeracy Studies, vol 16, no 1, pp 63 75.

Street, B and Baker, D (2006) So, What about Multimodal Numeracies? in Roswell, Jennifer and Pahl, Kate, eds, Travel Notes from the New Literacy Studies, Multilingual Matters Ltd, Cleveden, Buffalo, Toronto, pp 219-233.

Swales, J (2004) Research Genres. Explorations and Applications, Cambridge University Press, Cambridge. doi: http://dx.doi.org/10.1017/CBO9781139524827

Thesen, L (2014) Risk as Productive: Working with Dilemmas in the Writing of Research in Thesen, Lucia and Cooper, Linda, eds, Risk in Academic Writing. Postgraduate students, their teachers and the making of knowledge, Multilingual Matters, Bristol, Buffalo, Toronto, pp $1-26$.

Tufte, ER (2001) Second Edition. The Visual Display of Quantitative Information, Graphics Press, Cheshire, Conn.

Van Leeuwen, T and Jewitt, C, eds, (2001) Handbook of Visual Analysis, SAGE Publications, London, Thousand Oaks, New Dehli. 
